\title{
L'indicialisation ou la communication saisie par le nombre
}

Alain Cubertafond

\section{OpenEdition}

\section{Journals}

Édition électronique

URL : https://journals.openedition.org/communicationorganisation/2461

DOI : 10.4000/communicationorganisation.2461

ISSN : 1775-3546

\section{Éditeur}

Presses universitaires de Bordeaux

\section{Édition imprimée}

Date de publication : 1 novembre 2000

ISSN : 1168-5549

Référence électronique

Alain Cubertafond, «L'indicialisation ou la communication saisie par le nombre », Communication et organisation [En ligne], 18 | 2000, mis en ligne le 27 mars 2012, consulté le 05 août 2021. URL : http:// journals.openedition.org/communicationorganisation/2461 ; DOI : https://doi.org/10.4000/ communicationorganisation.2461

Ce document a été généré automatiquement le 5 août 2021 .

(C) Presses universitaires de Bordeaux 


\title{
L'indicialisation ou la communication saisie par le nombre
}

\author{
Alain Cubertafond
}

Dans le vaste espace de réflexion ouvert par le Groupe de Recherche en Communication des Organisations autour du non-verbal, il se trouve peut-être une forme transversale d'induction, que j'appelle indicialisation, qui me paraît occuper un espace de plus en plus large dans les systèmes modernes de communication.

2 L'indicialisation tient à la place et au rôle du quantifiable (l'arithmétique) puis du calculable (l'algèbre). Elle fonctionne sur des opérations mathématiques (équations, inéquations, ratios, logarithmes, algorithmes, exponentielles, etc.) réalisées au moyen des symboles représentant la mesure, les chiffres et les nombres. Elle s'exprime, généralement en termes de probabilités, par des taux, des pourcentages, des moyennes, des écarts, des coefficients de corrélations ou de fréquences. Elle se développe grâce à la progression incessante de la puissance et des vitesses de calcul, sur fond d'augmentation des capacités de mémoire de machines toujours plus performantes.

3 Comme chacun sait, le nombre est à la fois cardinal parce qu'il dit la quantité (du singulier au multiple) et ordinal parce qu'il dit l'ordre (du semblable au différent). Mais on n'a peut-être pas encore suffisamment montré que le nombre marque la communication d'une trace plus importante encore, même si, ce faisant, on s'expose à la critique des sciences "dures", relancée il y a quelques années par Alan Sokal. Traceur non-verbal ou, plus poétiquement, "lecture sourde » et " écriture muette ", pour reprendre ce que disait Hegel à propos des hiéroglyphes ${ }^{1}$ » le nombre forme pléthore de signes et de dispositifs communicants.

4 Chacun sait également que le nombre et ses dérivés sont inséparables des technologies et des réseaux de communication, où les codes numériques des signaux, données, images et sons ont facilement réussi à supplanter les procédés analogiques. Le numérique a également investi jusqu'à une position devenue centrale les outils professionnels et individuels de l'activité communicationnelle (production, transmission, diffusion, stockage, exploitation) grâce au développement d'une large palette de nouveaux dispositifs communicants (traitement de textes, images virtuelles, 
synthèse vocale, Internet, lecture et écriture numérique...) qui accompagnent l'émergence de nouvelles pratiques, souvent marchandes, de communication (e business, émail, e-book, home-cinema....).

Bien qu'elle soit pour toutes ces raisons généralement présentée comme une révolution dans la communication, la numérisation demeure encore souvent analysée dans les limites somme toute étroites d'une technologie capable de produire à la fois de nouveaux outils et des usages inédits. Car, paradoxalement, en donnant abondamment où et avec qui communiquer les impressionnants progrès des dispositifs numériques limitent la communication à ce qu'il est possible d'exprimer par le langage et les liaisons balisées des standards hypertextuels (HTLM ou hypertext mark up language et http, ou hypertext transfer protocol), au moyen des signes compressés des codes de l'image (MPEG ou moving picture expert group) ou du son (MP3) et dans un vocabulaire simplifié (comme l'alphabet simplifié des smileys ou les dictionnaires de quelques centaines de mots pour toute une langue). En devenant données, la parole, le texte, les sons ou l'image laissent aux signes de la mesure le soin de construire avec l'efficacité requise pour s'assurer d'être compris tant la forme globale des discours (politique, économique et médiatique) que, de façon plus concrète, l'expression des faits et des récits de la communication.

Les systèmes contemporains de communication se trouvent ainsi saisis par la discrète, voire secrète, polarité d'une indicialisation généralisée qui, parce qu'elle transpose et élargit les traditionnelles applications clients serveurs - autrement dit faire " tourner " les données du client sur le serveur - pourrait se révéler capable de prendre en charge de larges pans de l'activité communicationnelle.

On en observera les manifestations à trois niveaux concentriques qui s'élargissent progressivement du terrain des sondages d'audience et d'opinion au discours pour tenter, en fin de compte, de s'incarner dans l'individu devenu « homme numérique $»^{2}$.

\section{Sondages : de l'observation à l'interprétation.}

8 C'est à la base autour de la recherche des effets de la communication sur l'opinion et les attitudes des gens, autrement dit de la mesure de son audience, naguère chasse gardée des travaux théoriques et pratiques de la sociologie, de la linguistique ou de la sémiologie, que se sont enracinés les signes du calcul dans l'activité communicationnelle.

9 Sur cette voie, ouverte il y a plus d'un demi-siècle, loin du verbal, autour des opérations de saisie, d'enregistrement et de traitement de l'information produite par les sondages, s'est développée une nouvelle métrologie chargée de mesurer, trier, classer, croiser, exposer, analyser, la masse énorme des chiffres qui remontent en permanence du terrain obscur et complexe des faits de société. Les stratèges de l'opinion, du marketing ou des médias ont compris les premiers que le calcul des données saisies par les sondages se prêtait parfaitement à "gérer la multiplicité des événements engendrés par la multiplicité des singularités $»^{3}$. Ils sont désormais parvenu à utiliser efficacement le nombre pour identifier des familles de comportements, réaliser des profils de consommateurs, d'électeurs ou de spectateurs et d'interpréter l'état d'esprit du public, afin de lui proposer de façon très concrète les produits et services qu'il peut désirer au moment où il en a besoin de même que la publicité qui les accompagne. 

traditionnels d'opinion (notables, éditorialistes, professeurs, directeurs de conscience...) s'est rapidement révélée moins efficace que les batteries d'indices produites par les protocoles scientifiques du calcul devenues, qu'on le veuille ou non. tout à la fois : le principal appareil d'enregistrement et d'analyse des faits sociaux, une source essentielle d'information sur les comportements, et une part de plus en plus grande de la mémoire collective.

11 Forts de leurs protocoles mathématiques, les sondages ont depuis longtemps dépassé le stade $\mathrm{du}$ classement statistique $\mathrm{du}$ chaos proliférant des opinions ou des comportements pour tenter d'y mettre de l'ordre. Ce passage scientifiquement hasardeux de l'observation à l'interprétation procède vraisemblablement de l'idée selon laquelle le réel peut surgir du calcul lorsqu'on s'y emploie avec des méthodes appropriées: sommations de plus en plus fines, typologies, hiérarchies, affinités, symétries ou oppositions, redressement de moyennes, probabilités, analyses factorielles, structuration des données.

exemples peuvent être rapidement évoquées pour illustrer la diversification des applications quantitatives et qualitatives des sondages. Celui de la constitution de banques de données, l'information étant collectée en partie directement par de volumineux questionnaires, mais également à partir d'annuaires, de fichiers d'adresses, d'achats, d'abonnements, d'assurances ou encore de plus en plus, par les traces informatiques laissées par l'utilisation de dispositifs numériques. Le croisement de tous ces fichiers qui sont vendus, loués ou échangés constitue les méga-bases de données d'un ultra-çiblage jugé indispensable pour communiquer efficacement. Un second exemple, qualitatif, témoigne de l'intérêt soutenu des sondages pour le non-verbal, celui des calculs d'affinité, largement utilisés par le mediaplanning, désormais complétés par la détection d'émotions par mesure des réactions sensorielles. Plusieurs approches de mesures d'implications ${ }^{4}$ sont d'ores et déjà au catalogue de sociétés américaines de recherche en marketing.

Derrière l'extrême sophistication de ces opérations de comptage et de calcul se profile un projet d'ethnographie informatisée beaucoup plus ambitieux que celui de l'analyse systémique parce qu'il qui dévoile le détail des attitudes des gens, pose le diagnostic permanent de leurs comportements, en montre les évolutions et cherche à anticiper sur leurs réactions. Au fil d'un processus désormais continu et capable de fonctionner en temps réel sur Internet, les représentations et la jouissance que produisent les systèmes de communication peuvent être ordonnées, contrôlées et orientées, pour l'essentiel vers des relations marchandes, tant il est vrai que « qui manie le miroir tient l'homme à sa merci $»^{5}$.

\section{Discours : du déchiffrage au chiffrage.}

Parce qu'il est capable d'absorber la complexité en maîtrisant la panique qui surgit de la prolifération des possibles, que les bases de données qu'il fait "tourner » lui permettent non seulement de déchiffrer mais également de chiffrer voire de crypter, le nombre peut donner forme à la grille de différents discours. Il peut également en fournir les clés, au sens des chiffres des codes nécessaires à la reconnaissance mutuelle, ce que sont sous d'autres signes, les clés de la fiction littéraire. Après l'art et la littérature, sur nos écrans, le nombre surgit du chaos ténébreux de l'expérience, pour 
tenter de dire le contenu de l'échange symbolique ou, au moins, d'en fixer l'incertitude et l'instabilité foncières. En tirant au clair le réel par ses chiffrages, le nombre communique « car on ne communique pas dans les ténèbres où chacun se tient en tant qu'individu mais en partageant ce qu'on a en commun $»^{6}$. De fait, il se trouve étroitement impliqué dans la production du discours politique comme dans celle de bon nombre des récits de l'information et de la publicité proposés par les médias.

me parait ni étonnant ni scandaleux que l'abondance et la richesse des données ainsi récoltées sur un terrain sans cesse labouré par la métrologie des sondages puisse faire l'objet d'un recyclage. Il ne s'agit pas ici d'évoquer l'utilisation analogique ou métaphorique des concepts et des outils des sciences exactes dans les sciences humaines. Il s'agit d'observer que le calcul ne s'investit pas seulement dans le pilotage des effets de la communication mais qu'il entre dans la construction de nouvelles mythologies naturalistes, dont les récits dé sublimés surdéterminent les discours de la communication de masse.

La politique, comme espace de gestion des affaires communes dans le respect des libertés individuelles, est sans cesse confrontée à la pluralité des opinions et à la violence des oppositions. Dans cet espace, lien social est resserré en continu grâce aux coagulations statistiques produites à partir des sondages fonctionnant comme langage de l'opinion avec la caution scientifique du calcul. À titre limitatif, on peut en observer les scénarios :

17 - dans la régulation de l'opposition privé/public autour d'une définition

18 minimale des libertés individuelles,

19 - dans la modélisation des phénomènes d'opinion pour en éliminer les dérives,

20 - dans le formatage d'effets d'annonce pour optimiser les possibles

21 - ou, plus subtilement encore, dans la programmation anticipée de l'actualité. L'exemple le plus réussi à cet égard est sans doute, l'épisode fameux de la poignée de main entre Yasser Arafat et Itzhac Rabin, mise en scène sur CNN en direct de la Maison blanche par le président Clinton en 1993.

Quant aux médias, dont les profits sont directement liés au développement de leur audience, ils sont passés maîtres dans l'exploitation du nombre. Ils parviennent à la fois à la mise en indice généralisée de leur public à l'intention des publicitaires et des annonceurs et à optimiser leurs programmes sous couvert de refléter les courants qui parcourent le corps social. Dans cet exercice, une attention particulière est portée tant à la réalité brute des lieux, des événements et du vécu des gens qu'à la mise en scène publique du privé grâce aux photos des paparazzi, aux jeux, aux reality-shows, séries ou sitcoms... Le tout étant servi par les nouveaux experts de la médiation, sondeurs programmateurs, publicitaires et présentateurs mais aussi mediaplanners, rédacteurs concepteurs, attachés de presse ou encore scénaristes, stylistes, agences de casting, décorateurs et désormais webmasters.

Car, grâce aux codes et aux standards numériques du multimédia et d'Internet, les serveurs deviennent émetteurs en se chargeant, au delà de fonctionnalités visuelles et/ ou auditives, de protocoles sémantiques et documentaires. Les réseaux balisent plus nettement encore leurs discours que les médias traditionnels parce qu'ils fonctionnent en permanence en temps réel. Et ils évacuent à leur tour l'imagination lorsqu'ils recomposent en formes binaires, ludiques et résumées la piste de tous les savoirs, en les 
découplant de leurs supports traditionnels, papier, bande magnétique ou supports chimiques.

\section{Individu : cérébralisation ou crétinisation ?}

Au-delà du formatage de l'audience ou de l'opinion et du chiffrage des récits, la métrologie du calcul informatisé progresse inexorablement vers un individu «branché » mais isolé, ciblé, fiché et déjà précodé par ses numéros de carte bancaire, de sécurité sociale ou de téléphone. Car, à mesure que les limites de l'incalculable reculent sous l'effet de la fantastique progression du pouvoir - c'est-à-dire de la capacité d'agir - du calcul, on assiste à la disparition de l'incommensurable et par conséquent, au-delà de la socialisation des récits, à la possibilité de rendre objective toute subjectivité.

Un "cerveau collectif», selon l'expression de Joël de Rosnay, serait sur le point de surgir des protocoles statistiques ou mathématiques que mettent en œuvre les logiciels tandis que l'interconnexion des réseaux pourrait concurrencer les formes traditionnelles de l'organisation. Son intelligence serait capable de soumettre l'infinie richesse du débat, de l'imagination ou des opinions à l'ordre des calculs de probabilités qui, parce qu'ils présentent l'avantage de mesurer l'incertitude, forment à mesure qu'ils se généralisent une conscience de la certitude. Il se joue un tour semblable du côté du visuel recomposé par les standards numériques d'échantillonnage, de quantification et de compression de l'image virtuelle.

"Cérébralisation" ou "crétinisation»? A cette questions qu'aimait poser Gilles Deleuze $^{7}$ répondent les mises en garde déjà lointaines de Descartes qui écrivait à Fermat « je considère tout ce qui n'est que probable comme presque faux » ou celle du mathématicien René Thom qui explique que «si le calcul est fondé sur des éléments statistiques qui ne sont donc pas numériquement précis... la théorie des probabilités est fondamentalement une imposture $»^{8}$.

Quant l'intelligence artificielle des microprocesseurs ou des logiciels entre dans la moindre des machines communicante comme le " concept » est entré dans les produits et les services, à quoi bon penser? Les logiciels de Microsoft qui équipent $90 \%$ des ordinateurs de la planète pensent pour nous. Dans un tel contexte, la pensée, déclarée incommunicable, se trouverait marginalisée tandis que le détour par l'abstrait serait réservé à l'art - qui y a fait ses preuves - et que l'histoire ou la mémoire seraient balayées par l'actualité.

On comprend qu'il soit devenu tentant sans prendre beaucoup de risques, l'ordre étant extrait du chaos statistique, de substituer l'indice au point de vue, à l'expérience, au dialogue, au témoignage. Reste que - la mesure des choses n'étant pas les choses même si elle participe à leur connaissance - il faut regarder du côté des dérives dont est porteuse la logique d'incarnation de ce processus numérique d'objectivation. La plus évidente est celle de "déverbaliser " la relation devenue indiscutable sous l'emprise des nombres ; la plus grave - la conscience étant réduite au calcul - est sans doute celle d'assimiler l'indicialisation à un processus de type démocratique; la plus perverse est peut-être d'être devenue si communément admise par tous qu'elle est sur le point d'être individuellement intériorisée. 


\section{NOTES}

1. Dans l'Encyclopédie des sciences philosophiques de 1817.

2. Nicolas Negroponte. L'homme numérique, Robert Laffont, 1995.

3. Pascal Robert, "Qu'est-ce qu'une technologie intellectuelle», Communication et Langages, $\mathrm{n}^{\circ} 123,1^{\mathrm{er}}$ trimestre 2000

4. La recherche en marketing invente le détecteur d'émotions, Le Monde. 18/04/2000 reprenant le New York Times du 13/04/2000.

5. Pierre Legendre, La fabrique de l'homme occidental. Editions Mille et une nuits/Arte Editions, 1996.

6. Entretien avec Marie-José Mondzain, Le Monde, 8 sept. 1998.

7. Cf. notamment Gilles Deleuze, "Sur l'image-temps ", entretien dans Cinéma, n 334,dec. 1985.

8. Un entretien avec René Thom, «La recherche scientifique délaisse les grandes question théoriques », Le Monde, 22/23 avril 1995.

\section{AUTEUR}

\section{ALAIN CUBERTAFOND}

Université de Limoges 